\title{
Leukoencephalopathy with Cognitive Impairment following Tocilizumab for the Treatment of Rheumatoid Arthritis (RA)
}

\author{
Katsuya Kobayashi ${ }^{1}$, Yoko Okamoto ${ }^{1}$, Haruhisa Inoue ${ }^{1}$, Takashi Usui ${ }^{2}$, Masafumi Ihara ${ }^{1}$, \\ Jun Kawamata ${ }^{1}$, Yukio Miki ${ }^{3}$, Tsuneyo Mimori ${ }^{2}$, Hidekazu Tomimoto ${ }^{4}$ \\ and Ryosuke Takahashi ${ }^{1}$
}

\begin{abstract}
The biological agent tocilizumab, is a humanized, anti-human interleukin-6 receptor antibody. A 72-yearold woman developed cognitive impairment during the Phase III clinical trial of tocilizumab for the treatment of rheumatoid arthritis. MRI demonstrated hyperintense dissemination throughout the white matter on T2WI. An initial diagnosis of possible progressive multifocal leukoencephalopathy was made, but the PCR for JC virus DNA was negative in the CSF. The leukoencephalopathy might have been caused by a mechanism related to tocilizumab itself. It is strongly recommended to perform MRI if a patient develops any cognitive impairment during tocilizumab therapy.
\end{abstract}

Key words: rheumatoid arthritis, tocilizumab, leukoencephalopathy, cognitive impairment, MRI, interleukin 6

(Inter Med 48: 1307-1309, 2009)

(DOI: 10.2169/internalmedicine.48.1926)

\section{Introduction}

Tocilizumab is a humanized, anti-human interleukin-6 receptor antibody, produced by genetic engineering technology. To date, it has been prescribed solely to patients with Castleman disease. Phase III clinical trials of tocilizumab have already been performed on patients with rheumatoid arthritis. Here, we report the first case of leukoencephalopathy with cognitive impairment, likely caused by tocilizumab for the treatment of rheumatoid arthritis.

\section{Case Report}

A left-handed 72-year-old woman was referred to our hospital because her cognition had worsened over the previous two months. She had suffered from rheumatoid arthritis (RA) for the past six years, and since a daily dosage of 10 mg prednisolone had not been proved effective, it was decided that she participated in the Phase III clinical trial of tocilizumab. During the trial, the patient took $8 \mathrm{mg} / \mathrm{kg}$ of tocilizumab every four weeks. Forty months after commencing to take tocilizumab, she began to show signs of dementia and abnormal behavior. She became unable to check blood glucose by herself, put things in order, and speak coherently.

At the time she was admitted, her blood pressure was $124 / 64 \mathrm{mmHg}$, and her body temperature was $37.2^{\circ} \mathrm{C}$. A physical examination revealed bilateral pretibial edema and ankylosis of several joints. She was orientated, but anxious and nervous. She was not able to concentrate, and showed cognitive dysfunction with a Mini-Mental State Examination (MMSE) score of 23 and a Frontal Assessment Battery (FAB) score of 8. Snout reflex and bilateral sucking reflexes were positive. There were no motor or sensory disturbances. Bilateral Achilles tendon reflexes were decreased. Laboratory investigation demonstrated normal blood cell counts.

\footnotetext{
${ }^{1}$ Department of Neurology, Kyoto University Hospital, Kyoto, ${ }^{2}$ Department of Rheumatology and Clinical Immunology, Kyoto University Hospital, Kyoto, ${ }^{3}$ Department of Diagnostic Imaging and Nuclear Medicine, Kyoto University Hospital, Kyoto and ${ }^{4}$ Department of Neurology, Mie University Hospital, Tsu

Received for publication December 7, 2008; Accepted for publication March 22, 2009

Correspondence to Dr. Yoko Okamoto, yoko416@kuhp.kyoto-u.ac.jp
} 


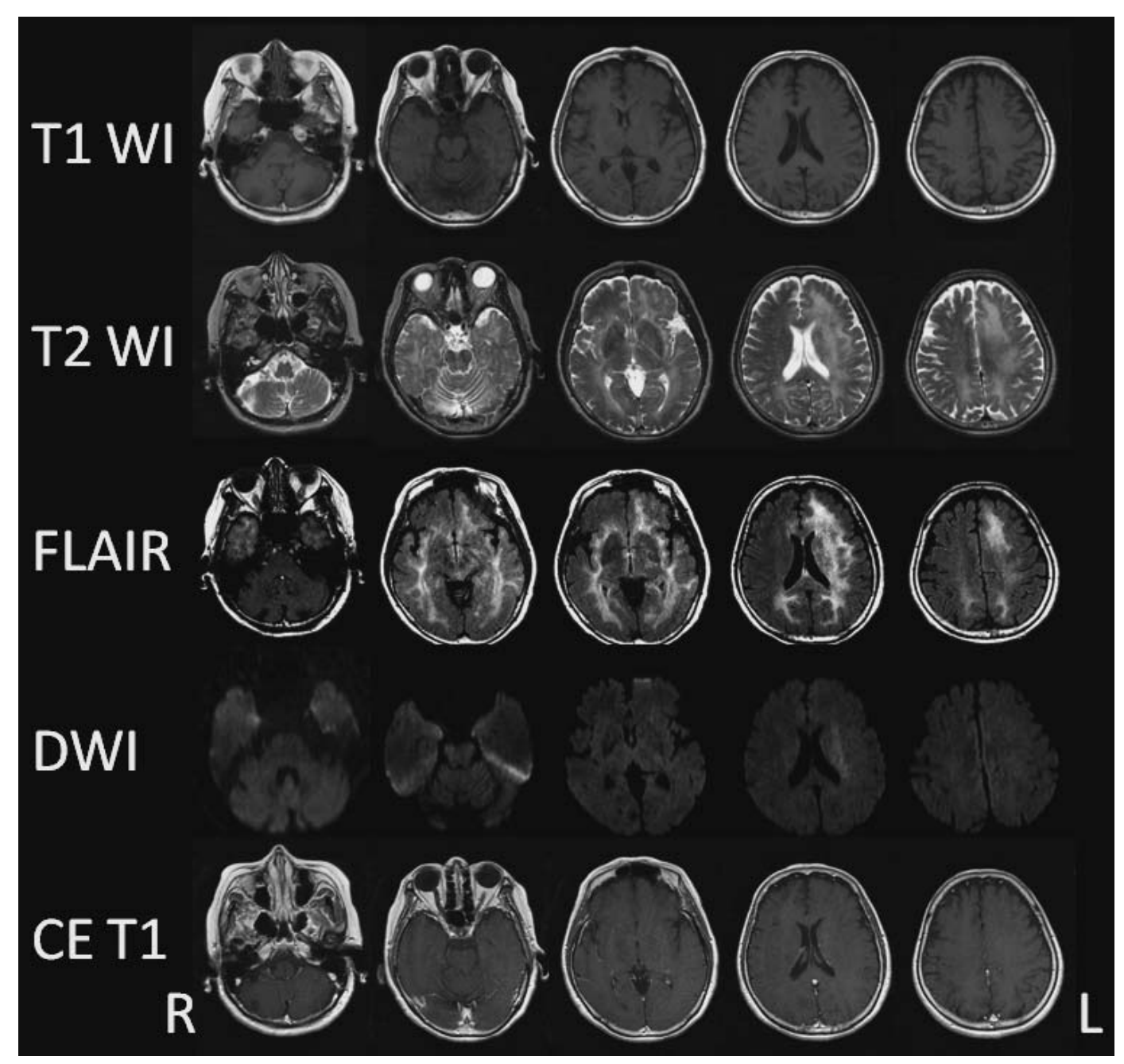

Figure 1. Brain MRI at the time of the admission. MRI disclosed high intensity dissemination throughout the white matter which spread widely from the temporal tip to the pars opercularis on T2WI and FLAIR. In accordance with the high intensity lesions on T2WI and FLAIR, slightly low intensity lesions were found on T1WI. There was no high intensity lesion on DWI except for T2 "shine-through" lesions of the left corona radiata. There was no mass effect or contrast enhancement lesion on contrast-enhanced T1WI.

Serum biochemistry showed an elevation of HbA1c (6.2\%), anti-nuclear antibody, rheumatoid factor (RF, 62.3 IU/mL) and matrix metalloprotainase-3 (MMP-3, $215 \mathrm{ng} / \mathrm{mL}$ ). The other autoimmune antibodies including anti-double stranded DNA, ribonucleoprotein, SS-A, SS-B, Scl-70, Jo-1, cANCA, p-ANCA, thyroid peroxidase (TPO), and thyroglobulin (TG) antibodies were all negative. Anti HTLV-I and HIV antibodies were also negative. Thyroid hormone, vitamins, angiotensin-converting enzyme (ACE), and antisoluble interleukin 2 receptor antibodies were within normal limits. The cerebrospinal fluid (CSF) revealed a leukocyte count of $2 / \mu \mathrm{L}$, a protein level of $46.6 \mathrm{mg} / \mathrm{dL}$ and a glucose level of $61 \mathrm{mg} / \mathrm{dL}$. IgG index elevated to 0.77 and a polymerase chain reaction for JC virus DNA was negative in the CSF.

A brain CT scan demonstrated diffuse low density in the bilateral cerebral white matter. An MRI disclosed high intensity disseminated throughout the white matter which spread widely from the temporal tip to the pars opercularis (Fig. 1).
There was no mass effect or contrast enhancement. On the scout view film of the cervical CT checked 6 months earlier, the low density area in the temporal tip and the frontal white matter was already present.

The background activity of electroencephalography consisted of 8-9 Hz, which suggested moderate dysfunction with left dominance. SPECT showed decreased uptake in accordance with the lesions observed in MRI. Although tocilizumab had been discontinued for the previous 5 months, her cognitive function and MRI findings did not change.

\section{Discussion}

The patient developed cognitive impairment in the course of tocilizumab therapy for RA. There are various pathoetiologies which may cause diffuse leukoencephalopathy, including collagen disease, vasculitis, reversible posterior leukoencephalopathy syndrome (RPLS), Binswanger disease, isolated angiitis of the central nervous system (CNS), pro- 
gressive multifocal leukoencephalopathy (PML), malignant lymphoma, gliomatosis cerebri, and radiation necrosis (1). The serum autoimmune antibodies examined were all negative and the JC virus DNA in the CSF was negative. The MRI showed asymmetrical white matter lesion and no mass effect, contrast enhancement, or cortical lesions. Thus, we could not obtain any supportive evidence for the diagnosis except the probable relevance with tocilizumab. However, the possibility of infection could not be entirely excluded during the hospitalization; therefore immunological therapy was not given.

Drug-induced leukoencephalopathy has been reported on amphotericin B, acyclovir, methotrexate, fluorouracil, tacrolimus, ciclosporin, etc (2). Also, biological agents, such as etanercept, infliximab, rituximab, or natalizmab cause leukoencephalopathy (3-6). Therefore, we postulated that tocilizumab caused the leukoencephalopathy via immunological mechanisms relating to direct and/or indirect IL-6 signaling. When we consider the concrete mechanism, this patient was aged, hypertensive, and undergoing steroid therapy. These factors might cause a synergistic effect on blood brain barrier (BBB) leading to leukoencephalopathy. Once BBB is disrupted, tocilizumab may also spread to the brain parenchyma and suppress IL-6 related glial survival. In fact, direct injection of IL- 6 into the brain can reduce ischemic brain injury, though its mechanism is unknown to date (7).

It remains unknown why the leukoencephalopathy was not resolved after tocilizumab discontinuation. One of the plausible explanations is that the leukoencephalopathy had been so advanced and irreversible that symptomatic relief was not obtained. As mentioned above, because the patient was aged, hypertensive, and undergoing steroid therapy, these backgrounds might have some effects on her irreversible symptoms. The pathological changes might be irreversible demyelination or chronic ischemic changes; however, further study is necessary.

This is the first report regarding the development of leukoencephalopathy during the administration of tocilizumab. Therefore, during tocilizumab therapy, it is strongly recommended to conduct a brain MRI if the patient develops any cognitive impairment.

\section{References}

1. Hinchey J, Chaves C, Appignani B, et al. A reversible posterior leukoencephalopathy syndrome. N Engl J Med 334: 494-500, 1996.

2. Shimono T, Miki Y, Toyoda H, et al. MR imaging with quantitative diffusion mapping of tacrolimus-induced neurotoxicity in organ transplant patients. Eur Radiol 13: 986-993, 2003.

3. Yamamoto M, Takahashi H, Wakasugi H, et al. Leukoencephalopathy during administration of etanercept for refractory rheumatoid arthritis. Mod Rheumatol 17: 72-74, 2007.

4. Roos JC. Neurological complication of infliximab. J Rheumatol
34: 236-237, 2007

5. US Food and Drug Administration. FDA alert: rituximab (marketed as Rituxan). December 2006.

6. Van Assche G, Van Ranst M, Sciot R, et al. Progressive multifocal leukoencephalopathy after natalizumab therapy for Crohn's disease. N Engl J Med 353: 362-368, 2005.

7. Suzuki S, Tanaka K, Suzuki N. Ambivalent aspects of interleukin6 in cerebral ischemia: inflammatory versus neurotrophic aspects. J Cereb Blood Flow Metab 29: 464-479, 2009.

(C) 2009 The Japanese Society of Internal Medicine http://www.naika.or.jp/imindex.html 